\title{
Pilot Study to Test the Utility and Acceptability of An Electronic Health Record (patientMpower) for Patients With Lung Fibrosis
}

\author{
Colin Edwards ${ }^{1}$, PhD; Nicola Cassidy ${ }^{2}$; Eamonn Costello ${ }^{1}$; Anne-Marie Russell ${ }^{3}$ \\ ${ }_{1}$ patientMpower Ltd., Dublin, Ireland \\ ${ }^{2}$ Irish Lung Fibrosis Association, Dublin, Ireland \\ ${ }^{3}$ Royal Brompton Hospital, London, United Kingdom
}

\section{Corresponding Author:}

Colin Edwards, PhD

patientMpower Ltd.

Digital Depot

Thomas Street

Dublin,

Ireland

Phone: 353872599131

Email: colin@patientmpower.com

\section{Abstract}

Background: Idiopathic pulmonary fibrosis (IPF) is an irreversible condition associated with progressive dyspnoea, fatigue, cough and psychological distress. Progression of IPF impacts physical activities affecting quality of life. Forced vital capacity (FVC) is the internationally accepted spirometry marker of disease progression. The patientMpower platform promotes supported self-management, enabling patients with IPF to record relevant objective and subjective measurements and health outcomes.

Objective: Assess patients' views on the utility and acceptability of the patientMpower platform in lung fibrosis.

Methods: Thirteen patients with lung fibrosis participated in a prospective, single-arm observational study (6 weeks). Invitations were issued through the Irish Lung Fibrosis Association. There were no changes to usual healthcare. Patients independently installed the patientMpower platform to their own smartphone/tablet. Technical support was available if needed. All patients were supplied with a Spirobank Smart spirometer (MIR, Rome, Italy) to record FVC at home. Instruction on correct use of the spirometer was given, supported by instructional video content within the platform. Patients were asked to use the patientMpower platform regularly, ideally daily. Measurements included: modified Medical Research Council breathlessness scale (mMRC), medication compliance, daily spirometry (seated), step count and weekly impact of lung fibrosis on daily life [Patient Reported Outcome Measure (PROM)]. Patients' opinions on utility and acceptability were assessed by a 17-point questionnaire.

Results: 13 patients ( 7 male; 54\%) participated. Median age: 66 years (range 37-83). Median baseline FVC: $78 \%$ predicted (range 40-123\%). Twelve patients (92\%) used the patientMpower platform for $\geq 6$ weeks and 8 (61\%) completed feedback questionnaires. $87 \%$ of patients $(n=7 / 8)$ who provided feedback questionnaires reported their experience of the platform as "positive" and stated they wanted to continue using it. To date, 8 patients $(61 \%)$, continue to actively use the platform after study completion. Nine patients $(69 \%)$ used the platform within 5 days of download and twelve used it on $\geq 60 \%$ of days over the observation period. Three patients $(23 \%)$ used it every day. Home spirometry was recorded frequently (median $69 \%$ of days; range $21-83 \%)$. mMRC data was infrequently recorded (0-7\% of days). Impact of lung fibrosis on daily life (PROM) was completed frequently (median $67 \%$ of weeks). At baseline, 12 patients $(92 \%)$ reported that breathing difficulties related to IPF affected their quality of life "some of the time" or "most of the time".

Conclusions: Patients with a severe life-limiting lung condition are willing and able to use an electronic health record to record objective data, symptoms and outcomes. Age is not a barrier to engaging with this technology. Regular home spirometry and recording of impact of lung fibrosis on daily life was feasible and acceptable in this patient population. A majority wished to continue using patientMpower indefinitely. This approach may be useful to capture patient-reported long-term trends in FVC and health outcomes.

(iproc 2017;3(1):e10) doi: 10.2196/iproc.8389

\section{KEYWORDS}

dyspnea; electronic symptom reporting; lung diseases; patient-reported outcomes; spirometry; IPF 


\section{Multimedia Appendix 1}

Full poster.

[PDF File (Adobe PDF File), 18MB-Multimedia Appendix 1]

Edited by T Hale; this is a non-peer-reviewed article. Submitted 10.07.17; accepted 23.08.17; published 22.09.17.

Please cite as:

Edwards C, Cassidy N, Costello E, Russell AM

Pilot Study to Test the Utility and Acceptability of An Electronic Health Record (patientMpower) for Patients With Lung Fibrosis iproc 2017;3(1):e10

URL: http://www.iproc.org/2017/1/e10/

doi: 10.2196/iproc.8389

PMID:

(C) Colin Edwards, Nicola Cassidy, Eamonn Costello, Anne-Marie Russell. Originally published in Iproceedings (http://www.iproc.org), 22.09.2017. This is an open-access article distributed under the terms of the Creative Commons Attribution License (https://creativecommons.org/licenses/by/4.0/), which permits unrestricted use, distribution, and reproduction in any medium, provided the original work, first published in Iproceedings, is properly cited. The complete bibliographic information, a link to the original publication on http://www.iproc.org/, as well as this copyright and license information must be included. 